\title{
PRODUÇÃO BIBLIOGRÁFICA: UMA ANÁLISE DE PESQUISAS PUBLICADAS NA REVISTA DE ADMINISTRAÇÃO DA UNIMEP - RAUNIMEP
}

\section{BIBLIOGRAPHIC PRODUCTION: A RESEARCH ANALYSIS PUBLISHED IN THE JOURNAL OF MANAGEMENT UNIMEP - RAUNIMEP}

\section{D0I: http://dx.doi.org/10.21714/raunp.v8i2.717}

\section{Silvia Helena Carvalho Ramos Valladão de Camargo}

Doutora e Mestre em Administração. Professora do Mestrado Profissional em Administração da Universidade Potiguar, Centro Universitário Moura Lacerda de Ribeirão Preto, Anhanguera Educacional Ltda. E-mail: shcamargo.ml@convex.com.br

\section{Dalila Alves Correa}

Doutora e Mestre em Administração. Docente e Pesquisadora do Programa de Pós-Graduação em Administração da Universidade Metodista de Piracicaba-UNIMEP. E-mail: dalvescorrea@gmail.com

\section{Graziela Oste Graziano}

Doutora e Mestre em Administração. Professora do Programa de Pós-Graduação Mestrado Profissional em Administração da Faculdade de Gestão e Negócios - Universidade Metodista de Piracicaba. E-mail: graziela.graziano@gmail.com

Envio em: Março de 2014

Aceite em: Abril de 2015

\section{RESUMO}

A construção e socialização do conhecimento científico é um dos pontos mais relevantes para os cenários econômico, científico e social de um país, é através desse processo que a Revista de Administração da UNIMEP (RAUNIMEP) vem se efetivando nos últimos anos como um periódico eletrônico bem-sucedido. 0 artigo teve como objetivo a análise da produção bibliográfica deste periódico veiculada no período de setembro de 2003 a agosto de 2012, através da coleta de dados secundários e de uma entrevista semiestruturada realizada com 0 seu editor. A pesquisa realizada mostra uma descrição da publicação do período apresentando informações sobre origem dos artigos, autoria, instituições participantes, as áreas e sub áreas dos artigos, dentre outros aspectos. Inclui considerações sobre o histórico do periódico e seu potencial de crescimento pelo nível de atendimento que vem obtendo dentro dos padrões Qualis CAPES nacionais e internacionais definidos para os periódicos científicos integrantes dos programas de Pós-graduação Strito Sensu.

Palavras-chave: Revista de Administração da UNIMEP. RAUNIMEP. Produção bibliográfica. Qualis CAPES. Administração.

\section{ABSTRACT}

The construction and socialization of scientific knowledge is one of the most relevant economic scenarios, scientific and social of a country, is through this process that the Revista de Administração da UNIMEP (RAUNIMEP) has been highlighted in recent years. The article aimed at analysis of bibliographic production RAUNIMEP from September 2003 to August 2012, through the collection of secondary data and a semistructured interview with your editor. The research shows considerations about publishing period presenting information about the origin of articles, authors, institutions, areas and sub areas of articles, among other aspects. Includes consideration of the history of the journal and its growth potential by the level of service that has achieved within the standards Qualis national and international CAPES set to scientific journals members of Graduate Strito Sensu programs.

Key-words: Revista de Administração da UNIMEP. RAUNIMEP. Bibliographic production. Qualis CAPES. Administration. 


\section{INTRODUÇÃO}

Desde a liberação comercial da internet, em 1995, a comunicação científica vem passando por transformações com o surgimento de novos canais e formatos que ampliaram a possibilidade de compartilhamento, bem como eliminação de barreiras geográficas (FERNANDES; CENDÓN, 2014).

Com o desenvolvimento da tecnologia de comunicação, especialmente computadores e redes eletrônicas, as formas de comunicação disponíveis à comunidade científica vêm se modificando, ampliando e diversificando, tornando-se cada vez mais eficientes, rápidas e abrangentes, vencendo barreiras geográficas, hierárquicas e financeiras. Essas mudanças estão ocorrendo tanto nos canais informais como nos formais. Dentre esses últimos, os mais importantes, para a Ciência, ainda são os artigos publicados em periódicos científicos impressos (MUELLER, 2000a, p. 23).

Isto se justifica diante das limitações que a comunicação científica impressa enfrenta para chegar até aos seus leitores. Dentre estas limitações mencionamos a demora na publicação do artigo; os elevados custos de aquisição e manutenção de coleções; a rigidez do formato impresso; a dificuldade, para o usuário, de recuperação da informação relevante; e a dificuldade, para o pesquisador, em ter acesso a trabalhos que são de seu interesse (MUELLER, 2000b).

É neste contexto de fortalecimento do periódico eletrônico que a Revista de Administração da UNIMEP - RAUNIMEP, locada no PPGA - Programa de Pós-Graduação em Administração, vem desenvolvendo desde 2003 a sua missão de "aprimorar e disseminar o conhecimento na área de administração" (RAUNIMEP, 2014).

A RAUNIMEP tem como foco a publicação de trabalhos de pesquisa, análises teóricas e resenhas sobre estratégia, organizações, marketing, gestão de pessoas, finanças, operações e logística.

Atualmente a revista está classificada como B1 no sistema Qualis/CAPES. Para se entender melhor como essa classificação é feita, é preciso primeiramente entender qual o papel da CAPES neste contexto. A Coordenação de Aperfeiçoamento de Pessoal de Nível Superior - CAPES foi criada em 1951 através de um decreto $\mathrm{n}^{\circ} 29.741$ com o objetivo de "assegurar a existência de pessoal especializado em quantidade e qualidade suficientes para atender às necessidades dos empreendimentos públicos e privados que visam ao desenvolvimento do país" (CAPES, 2014).

A CAPES possui um programa, o "Qualis periódicos", que tem como objetivo analisar a qualidade da produção intelectual dos programas de Pós-graduação. Essa análise é feita de forma indireta, ou seja, através da qualidade dos artigos e de outros tipos de produção e, a partir da análise da qualidade dos veículos de divulgação; os periódicos científicos (CAPES, 2014).

Periodicamente, esses veículos são avaliados e classificados conforme os indicativos de qualidade e, são classificados em uma escala que varia de A1 (o mais elevado nível de qualidade e com maior peso) a C (o mais baixo nível e de peso nulo), apresentando ainda as pontuações intermediárias: A2; B1; B2; B3; B4; B5 e C.

A RAUNIMEP tem publicações de autorias procedentes de todas as regiões brasileiras, além de internacionais oriundas de países como Argentina, México, Venezuela e Estados Unidos - são mais de 1200 usuários envolvidos nesse projeto, sendo eles: leitores e avaliadores (RAUNIMEP, 2012).

O objetivo desse artigo foi proceder uma análise da produção bibliográfica da RAUNIMEP publicada no período de setembro de 2003 a agosto de 2012, através de um estudo descritivo realizado sobre dados secundários, o qual inclui também uma entrevista realizada com o seu editor chefe. Procurou ainda apontar os objetivos futuros que a revista almeja nos próximos dez anos.

O artigo está estruturado em cinco seções: a partir da introdução, a revisão bibliográfica sobre o tema, a metodologia da pesquisa, a apresentação dos dados, discussão dos resultados e as considerações finais.

\section{DESCRIÇÃO DO QUALIS-PERIÓDICO}

O conjunto de procedimentos utilizados pela CAPES para a diferenciação da qualidade da produção bibliográfica dos programas de pós-graduação é chamado de Qualis.

O Qualis confere toda a qualidade dos artigos e de outros tipos de produção através da análise apurada da qualidade dos periódicos científicos, jornais, eventos, livros, etc.

Para a classificação dos veículos de divulgação da produção intelectual dos programas de Pós-graduação é utilizado o aplicativo WebQualis, que, além da 
classificação feita é também aplicado para a divulgação do resultado dessa classificação (através de uma lista por área) no site da CAPES (PLATAFORMA SUCUPIRA, 2016).

Essa classificação no Qualis é feita por comissões de consultores seguindo critérios diferenciados de cada conjunto de áreas específicas, os critérios variam conforme a área de atuação e o ano, sendo assim o processo de atualização do Qualis é anual.

A classificação dos periódicos é dividida em estratos indicativos de qualidade: A1; A2; B1; B2; B3; B4; B5; C.

A estratificação na área de Administração, Ciências Contábeis e Turismo (que é a área onde a Revista de Administração da UNIMEP se aloca) é atualizada anualmente pela comissão do Qualis-periódicos, a partir do fator de impacto dos periódicos consultados.

No Brasil, a concessão de financiamento de atividades científicas requer o estabelecimento de critérios na avaliação de pesquisadores e das instituições. Em meados dos anos 2000 o critério de avaliação da excelência acadêmica do pesquisador era quantitativo, hoje se leva em conta mais a qualidade das publicações do que a quantidade. Entretanto, a relação quali/quanti se estabelece em função da classificação da qualidade da produção e, não necessariamente da quantidade para se atingir uma pontuação mais elevada.

Uma das maneiras de avaliar a qualidade de uma publicação consiste em observar o grau de interesse dos outros pesquisadores por aquela pesquisa, e esta medida ocorre por meio da quantidade de citações dessa por outros autores em seus trabalhos.

Este processo de avaliação de qualidade é feito a partir do impacto das publicações na comunidade científica e é denominado no ramo da bibliometria ou cienciometria como análise de citações mais conhecido como Fator de Impacto (FI), e tem sido difundido mundialmente no âmbito das agências de fomento de pesquisa.

O Fator de Impacto (FI) "é um sistema que determina a quantidade de vezes que uma publicação é citada em certo período de tempo, dividida pela quantidade de artigos publicados nesse mesmo período. A Thomson Reuters, antigo Institute for Scientific Information mais conhecido como ISI utiliza nessa avaliação um período de dois anos". (BIREME, 2012).

A pontuação atribuída aos estratos na área de Administração, Ciências Contábeis e Turismo, segundo (WEBQUALIS, 2014) é a seguinte:

Estrato 7 - A1: 100 pontos

Estrato 6 - A2: 80 pontos

Estrato 5 - B1: 60 pontos

Estrato 4 - B2: 50 pontos

Estrato 3 - B3: 30 pontos

Estrato 2 - B4: 20 pontos

Estrato 1 - B5: 10 pontos

Estrato 0 - C: sem pontuação

O Quadro 1 demonstra os critérios de avaliação de um periódico, dentro de cada estrato.

\section{QUADRO 1: CRITÉRIOS DE AVALIAÇÃO DENTRO DE CADA ESTRATO}

\begin{tabular}{|c|c|}
\hline ESTRATO & CRITÉRIOS \\
\hline B5 & $\begin{array}{l}\text { Ter ISSN } \\
\text { Ter periodicidade definida }\end{array}$ \\
\hline B4 & $\begin{array}{l}\text { Atender as demandas para se enquadrar no estrato anterior } \\
\text { Ter revisões por pares } \\
\text { Edições atualizadas até } 2011 \\
\text { Normas de submissão }\end{array}$ \\
\hline B3 & $\begin{array}{l}\text { Atende as demandas para se enquadrar no estrato anterior } \\
\text { Atender aos } 6 \text { critérios abaixo: } \\
\checkmark \text { Missão/foco } \\
\checkmark \text { Informa o nome e afiliação do editor } \\
\checkmark \text { Informa o nome e afiliação dos membros do comitê editorial } \\
\checkmark \text { Divulga anualmente a nominata dos revisores } \\
\checkmark \text { Mínimo de dois números por ano } \\
\checkmark \text { Informa dados completos dos artigos } \\
\checkmark \text { Endereço de pelo menos um dos autores }\end{array}$ \\
\hline
\end{tabular}




\begin{tabular}{|c|c|}
\hline ESTRATO & CRITÉRIOS \\
\hline B2 & $\begin{array}{l}\text { Atende as demandas para se enquadrar no estrato anterior } \\
\text { Ter mais de três anos } \\
\text { Ter um indexador (SCOPUS, EBSCO, DOAJ, GALE, CLASE, HAPI, ICAP, IBSS) } \\
\text { Informações sobre os trâmites de aprovação } \\
\text { Apresentar a legenda bibliográfica da revista em cada artigo } \\
\text { Ter conselho diversificado } \\
\text { Editor chefe não é autor } \\
\text { Informação sobre o processo de avaliação }\end{array}$ \\
\hline B1 & $\begin{array}{l}\text { Atender aos estratos anteriores } \\
\text { Scopus e } 0<\mathrm{H} \text { Scopus }<4 \text { ou }<\mathrm{JCR}<0,2 \text {, o que for mais favorável ao periódico. } \\
\text { Ou } \\
\text { Estar na Scielo ou Redalyc } \\
\text { Ter mais de } 5 \text { anos } \\
\text { Ou } \\
\text { Ser periódico de uma das seguintes editoras: Sage; Elsevier; Emerald; Springer; Inderscience; Pergamo; } \\
\text { Wiley; e Routledge. }\end{array}$ \\
\hline A2 & $4<\mathrm{H}$ Scopus $<20$ ou $0,2<$ JCR $<1,0$, o que for mais favorável ao periódico. \\
\hline A1 & H Scopus $>20$ OU jcr $>1,0$, o que for mais favorável ao periódico. \\
\hline C & $\begin{array}{l}\text { Periódicos que não atendem os critérios para ser B5 ou não tiveram artigos da área neles publicados em } \\
\text { 2010. Periódicos que não tiveram publicação de artigo na área publicado em 2010, mas tenha em } 2011 \\
\text { ou 2012, serão qualificados num dos estratos acima, desde que atendam aos critérios estabelecidos. }\end{array}$ \\
\hline
\end{tabular}

Fonte: CAPES, 2012.

\section{METODOLOGIA DO ESTUDO}

Este é um estudo bibliográfico qualitativo e de natureza descritiva. Este tipo de investigação prioriza algumas técnicas empregadas para descobrir fenômenos latentes que reúnem um conjunto qualitativo de informações. De acordo com Chizzotti (1991), as técnicas que podem ser adotadas neste tipo de estudo podem ser desenvolvidas observando a vida cotidiana em seu contexto ecológico, ouvindo narrativas, lembranças e biografias, analisando documentos, obtendo-se um volume qualitativo de dados originais e relevantes não filtrados por conceitos operacionais e nem por índices quantitativos. O uso da estatística descritiva é adotado para possibilitar descrições e ilustrações.

$\mathrm{Na}$ pesquisa qualitativa a utilização destas técnicas não deve construir um modelo único, exclusivo e padronizado, ao contrário, deve "possibilitar a criação que mobiliza a acuidade inventiva do pesquisador, sua habilidade artesanal e perspicácia para elaborar a metodologia adequada ao campo da pesquisa, ao problema eleito" (CHIZZOTTI, 1991, p.81).

O estudo bibliográfico abrange a leitura, análise e interpretação de conteúdos de livros, periódicos, textos legais, documentos mimeografados ou xerocopia- dos, mapas, fotos, manuscritos etc. Refere-se a uma análise atenta e sistemática que se faz acompanhar de anotações e fichamentos. Seu objetivo é levar ao conhecimento das diferentes contribuições científicas disponíveis sobre determinado tema, além de oferecer contribuições para a evolução das análises críticas (GIL, 2002).

\subsection{PROCEDIMENTOS METODOLÓGICOS DO ESTUDO}

O estudo enquadra-se em uma perspectiva bibliométrica, onde "o termo bibliometria é utilizado para quantificar os processos de comunicação escrita e o emprego de indicadores bibliométricos para medir a produção científica (OLIVEIRA, 2001).

A pesquisa com a análise bibliométrica é utilizada para levantar comportamentos e incidências da literatura técnico-científica, como também sua evolução em contextos e épocas determinados (VANTI, 2002).

O universo da produção bibliográfica que compôs o estudo compreende as publicações de artigos científicos veiculados no periódico RAUNIMEP, Revista de Administração da Unimep, no período de 2003 a setembro de 2012.

Os referidos periódicos foram acessados através do 
site da revista (http://www.raunimep.com.br/ojs/index.php/regen/issue/archive, acesso em 23/09/2012).

Além da coleta de dados secundários, realizados no site da RAUNIMEP, foi realizada também uma entrevista semiestruturada com o editor chefe da mesma. Por entrevista semiestruturada, Gil (1999, p. 120) explica que "o entrevistador permite ao entrevistado falar livremente sobre o assunto, mas, quando este se desvia do tema original, esforça-se para a sua retomada". Para a análise dos dados utilizou-se o CiteSpace, software gratuito. As principais leis bibliométricas são: Lei de Bradford, (produtividade de periódicos), Lei de Lotka (produtividade científica de autores) e Leis de Zipf (frequência de palavras), ou Lei do mínimo esforço. A Bibliometria é também um instrumento quantitativo, que permite minimizar a subjetividade inerente à indexação e recuperação das informações, produzindo conhecimento em determinada área de assunto. Em última análise ela contribui para tomadas de decisão na gestão da informação e do conhecimento, uma vez que auxilia na organização e sistematização de informações científicas e tecnológicas.

\section{APRESENTAÇÃO E ANÁLISE DOS} DADOS COLETADOS

A coleta de dados envolveu as seguintes unidades de análises: ano de publicação dos artigos; região de origem; instituições de origem dos autores dos artigos e subáreas dos artigos. A seguir apresenta-se cada uma delas.

\subsection{NÚMERO DE ARTIGOS X ANO DE PUBLICAÇÃO}

O número de artigos publicado em cada edição da revista variou entre 8 a 30 artigos. O menor número ocorreu no ano de 2003 com 8 artigos (3,7\%). A maior publicação de artigos foi registrada em 2010 com 30 artigos (13,9\%). A média de artigos publicados é de 21 artigos por ano.

A Tabela 1 demonstra a distribuição da publicação dos artigos no período setembro/2003 a agosto/2012.

TABELA 1: PUBLICAÇÃO DE ARTIGO VERSUS ANO

\begin{tabular}{ccc}
\hline Ano & \multicolumn{2}{c}{ Artigos Publicados } \\
\hline & $\mathbf{F}$ & $\mathbf{f}(\%)$ \\
2003 & 08 & 3,7 \\
2004 & 23 & 10,5 \\
2005 & 23 & 10,5 \\
2006 & 23 & 10,5 \\
2007 & 24 & 11,1 \\
2008 & 21 & 9,7 \\
2009 & 24 & 11,1 \\
2010 & 30 & 13,9 \\
2011 & 24 & 11,1 \\
2012 & 17 & 7,9 \\
\hline Total & 217 & 100 \\
\hline
\end{tabular}

Fonte: RAUNIMEP, 2003 à 2012.

Nota: Além dos 217 artigos registrou-se também a presença de 12 artigos internacionais ao longo do período analisado. Estes não compõem as análises realizadas por opção de explorar apenas os artigos nacionais.

\subsection{PROCEDÊNCIA GEOGRÁFICA DOS ARTIGOS}

O número de artigos publicados na RAUNIMEP demonstra variações de acordo com a sua origem geográfica, a partir das autorias. Desse modo, observou-se que o maior número de artigos, do período analisado, origina-se de autores da região Sudeste $(57,20 \%)$. O menor número de artigos decorre de autorias da região Norte $(0,87 \%)$. Observou-se também que $5,27 \%$ dos artigos é de autores internacionais. A Tabela 2 demonstra o número de participações das regiões brasileiras e inclui também participações internacionais na RAUNIMEP. 
TABELA 2: PROCEDÊNCIA GEOGRÁFICA DOS ARTIGOS

\begin{tabular}{lcc}
\hline Região & Participações & $\mathbf{f ( \% )}$ \\
\hline Norte & 02 & 0,87 \\
Nordeste & 17 & 7,43 \\
Centro-Oeste & 04 & 1,75 \\
Sudeste & 131 & 57,20 \\
Sul & 63 & 27,51 \\
Internacional & 12 & 5,24 \\
\hline Total & 229 & 100 \\
\hline
\end{tabular}

Fonte: RAUNIMEP, 2003 à 2012.

\subsection{SUBÁREAS DOS ARTIGOS}

Os artigos foram publicados em 7 subáreas da Administração de Empresas, compreendendo: estratégia, finanças, gestão de pessoas, logística, marketing, operações e organização. A subárea com maior número de artigos $(23,5 \%)$ é a de estratégia, seguida de organização $(18,9 \%)$. O menor número de artigos está na subárea de logística $(4,6 \%)$.

A classificação das subáreas foi feita a partir da leitura e análise do título do artigo, das palavras-chave e de seus resumos. A Tabela 3 demonstra o número de artigos específicos em cada subárea.

TABELA 3: SUBÁREA VERSUS ARTIGOS ESPECÍFICOS

\begin{tabular}{lcc}
\hline Sub-área & Número de artigos específicos \\
\hline & $\mathbf{F}$ & $\mathbf{f}(\%)$ \\
Estratégia & 51 & 23,5 \\
Finanças & 22 & 10,2 \\
Gestão de Pessoas & 25 & 11,5 \\
Logística & 10 & 4,6 \\
Marketing & 36 & 16,6 \\
Operações & 32 & 14,7 \\
Organização & 41 & 18,9 \\
\hline Total & 217 & 100 \\
\hline
\end{tabular}

Fonte: RAUNIMEP, 2003 à 2012.

\subsection{INSTITUIÇÕES DE ORIGEM DOS AUTORES}

Os 217 artigos publicados no período setembro/2003 a agosto/2012 tem autores vinculados a 121 instituições de ensino superior nacional e internacional, compreendendo universidades, faculdades, institutos, centros universitários, fundações comunitárias, entre outras.

Registrou-se que a autoria de 32 artigos (14,74\%) se origina da Universidade de São Paulo (USP), seguida de 26 artigos (12\%) originários da Universidade Metodista de Piracicaba - UNIMEP.

Observou-se também a presença de autores de ins- tituições internacionais como: Universidad Madero (México) com 4 artigos (1,84\%); Universidad Autonoma de Tamaulipas (México), Universidad del Zulia (Venezuela), Marietta College (Estados Unidos), Universidad Autonoma de Sinaloa (México), Centro Universitario de Ciencias Económico Administrativas (México), Universidade Beira Interior (Portugal), Université Pierre Mendès (França), Universidad Nacional de La Plata (Argentina) e Universidad de Zaragoza (Espanha), cada instituição colaborou com 1 artigo $(0,46 \%)$.

A Tabela 4 demonstra todas as instituições de ensino superior de origem dos autores dos artigos publicados no período de setembro de 2003 à agosto de 2012. 
TABELA 4: INSTITUIÇÕES DOS AUTORES E PARTICIPAÇÕES

\begin{tabular}{|c|c|c|}
\hline $\begin{array}{l}\text { Instituição } \\
\text { com artigos } \\
\text { publicados }\end{array}$ & $\begin{array}{l}\text { Número de vezes que a } \\
\text { instituição participou com } \\
\text { artigos publicados }\end{array}$ & $f(\%)$ \\
\hline Universidade de São Paulo (USP - SP) & 32 & 14,74 \\
\hline Universidade Metodista de Piracicaba (Unimep - SP) & 26 & 12 \\
\hline Fundação Getúlio Vargas (FGV - SP) & 10 & 4,60 \\
\hline Universidade Federal de Santa Catarina (UFSC - SC) & 10 & 4,60 \\
\hline Universidade Nove de Julho (Uninove - SP) & 08 & 3,68 \\
\hline Universidade Federal de Minas Gerais (UFMG - MG) & 07 & 3,22 \\
\hline Universidade Presbiteriana Mackenzie (UPM - SP) & 07 & 3,22 \\
\hline Universidade Municipal de São Caetano do Sul (USCS - SP) & 06 & 2,76 \\
\hline Universidade Paulista (UNIP - SP) & 06 & 2,76 \\
\hline Universidade Federal de Lavras (UFLA - MG) & 06 & 2,76 \\
\hline Pontifícia Universidade Católica do Paraná (PUC-PR) & 06 & 2,76 \\
\hline Universidade Caxias do Sul (UCS - RS) & 06 & 2,76 \\
\hline Pontifícia Universidade Católica de São Paulo (PUC - SP) & 05 & 2,30 \\
\hline Universidade Estadual do Ceará (UECE - CE) & 05 & 2,30 \\
\hline Pontifícia Universidade Católica de Minas Gerais (PUCMinas - MG) & 04 & 1,84 \\
\hline Centro Universitário Moura Lacerda (SP) & 04 & 1,84 \\
\hline Universidad Madero (México) & 04 & 1,84 \\
\hline Universidade Federal da Paraíba (UFPB - PB) & 04 & 1,84 \\
\hline Universidade Federal do Rio Grande do Sul (UFRGS - RS) & 04 & 1,84 \\
\hline Universidade Regional de Blumenau (FURB - SC) & 04 & 1,84 \\
\hline Escola Superior de Propaganda e Marketing (ESPM - SP) & 04 & 1,84 \\
\hline Universidade Federal do Paraná (UFPR - PR) & 04 & 1,84 \\
\hline Universidade Positivo (Unicenp - PR) & 04 & 1,84 \\
\hline Instituto Brasileiro de Mercado de Capitais (Ibmec - RJ) & 03 & 1,38 \\
\hline Universidade Estadual Paulista "Júlio de Mesquita Filho"(UNESP - SP) & 03 & 1,38 \\
\hline Universidade Metodista de São Paulo (Umesp - SP) & 03 & 1,38 \\
\hline Universidade Federal de Santa Maria (UFSM - RS) & 03 & 1,38 \\
\hline Fundação Comunitária de Ensino Superior de Itabira (Funcesi - MG) & 03 & 1,38 \\
\hline Universidade Federal de Viçosa (UFV - MG) & 03 & 1,38 \\
\hline Universidade do Vale do Itajaí (Univale - SC) & 03 & 1,38 \\
\hline Instituto Mineiro de Educação Superior (IMES - MG) & 02 & 0,92 \\
\hline Universidade Cruzeiro do Sul (Unicsul - PR) & 02 & 0,92 \\
\hline Universidade Guarulhos (UNG - SP) & 02 & 0,92 \\
\hline Universidade Federal Rural do Rio de Janeiro (Ufrrj - RJ) & 02 & 0,92 \\
\hline Pontifícia Universidade Católica de Campinas (PUCCAMP - SP) & 02 & 0,92 \\
\hline Universidade Federal do Pernambuco (UFPE - PE) & 02 & 0,92 \\
\hline Universidade de Passo Fundo (UPF - RS) & 02 & 0,92 \\
\hline Universidade do Planalto Catarinense (Uniplac - SC) & 02 & 0,92 \\
\hline Universidade Estadual de Maringá (UEM - PR) & 02 & 0,92 \\
\hline Centro Universitário Franciscano (Unifra - RS) & 02 & 0,92 \\
\hline Universidade Federal de Uberlândia (UFU - MG) & 02 & 0,92 \\
\hline Universidade Federal do ABC (UFABC - SP) & 02 & 0,92 \\
\hline Universidade do Sul de Santa Catarina (Unisul - SC) & 02 & 0,92 \\
\hline Universidade do Estado de Santa Catarina (Udesc - SC) & 02 & 0,92 \\
\hline Universidade Federal da Grande Dourado (UFGD - MS) & 02 & 0,92 \\
\hline Faculdade Campo Limpo Paulista (Faccamp - SP) & 02 & 0,92 \\
\hline Universidade Estadual de Londrina (UEL - PR) & 02 & 0,92 \\
\hline Faculdade Adventista de Minas Gerais (FadMinas - MG) & 02 & 0,92 \\
\hline Faculdade de Orlândia (FAO-SP) & 01 & 0,46 \\
\hline Faculdade de Apucarana (FAP - PR) & 01 & 0,46 \\
\hline
\end{tabular}




\begin{tabular}{|c|c|c|}
\hline $\begin{array}{l}\text { Instituição } \\
\text { com artigos } \\
\text { publicados }\end{array}$ & $\begin{array}{l}\text { Número de vezes que a } \\
\text { instituição participou com } \\
\text { artigos publicados }\end{array}$ & $f(\%)$ \\
\hline Instituto de Estudos Superiores da Amazônia (IESAM - AM) & 01 & 0,46 \\
\hline Universidade Estadual de Campinas (Unicamp - SP) & 01 & 0,46 \\
\hline Universidade Estadual do Oeste do Paraná (Unioeste - PR) & 01 & 0,46 \\
\hline Faculdade do Norte Novo de Apucarana (Facnopar- PR) & 01 & 0,46 \\
\hline Universidade Federal da Bahia (UFBA - BA) & 01 & 0,46 \\
\hline Fundação Visconde do Cairú (FVC - BA) & 01 & 0,46 \\
\hline Faculdade São Paulo (FAC - SP) & 01 & 0,46 \\
\hline Universidade Camilo Castelo Branco (Unicastelo - SP) & 01 & 0,46 \\
\hline Universidade Católica Dom Bosco (UCDB - SP) & 01 & 0,46 \\
\hline Universidad Autonoma de Tamaulipas (México) & 01 & 0,46 \\
\hline Universidade Federal de Ouro Preto (UFOP - MG) & 01 & 0,46 \\
\hline Fundação Instituto de Administração (FIA - SP) & 01 & 0,46 \\
\hline Centro Universitário de Faculdades Associadas de Ensino(Unifae-PR) & 01 & 0,46 \\
\hline Universidade de Mogi das Cruzes (UMC - SP) & 01 & 0,46 \\
\hline Faculdades Metropolitanas Unidas (FMU - SP) & 01 & 0,46 \\
\hline Universidade Salvador (Unifacs - BA) & 01 & 0,46 \\
\hline Centro Universitário Assunção (Unifai - SC) & 01 & 0,46 \\
\hline Universidade Católica de Santos (Unisantos - SP) & 01 & 0,46 \\
\hline Fundação Pedro Leopoldo (Unipel - MG) & 01 & 0,46 \\
\hline Centro Universitário Salesiano de São Paulo (Unisal - SP) & 01 & 0,46 \\
\hline Universidad del Zulia (Venezuela) & 01 & 0,46 \\
\hline Faculdade Editora Nacional (Faenac - SP) & 01 & 0,46 \\
\hline Fundação Armando Álvares Penteado (FAAP - SP) & 01 & 0,46 \\
\hline Universidade Federal do Ceará (UFC - CE) & 01 & 0,46 \\
\hline Faculdade Integrada do Recife (FIR - PE) & 01 & 0,46 \\
\hline Faculdade de Administração da Associação Brasiliense de Educação (FABE - RS) & 01 & 0,46 \\
\hline Universidade do Vale do Rio dos Sinos (Unisinos - RS) & 01 & 0,46 \\
\hline Universidade do Vale do São Francisco (Univasf - BA) & 01 & 0,46 \\
\hline Pontifícia Universidade Católica do Rio Grande do Sul (PUC - RS) & 01 & 0,46 \\
\hline Universidade Federal do Pampa (Unipampa - RS) & 01 & 0,46 \\
\hline Faculdade Estadual de Ciências e Letras de Campo Mourão (Fecilcam - PR) & 01 & 0,46 \\
\hline Centro Universitário de Belo Horizonte (UniBH - MG) & 01 & 0,46 \\
\hline Marietta College (Estados Unidos) & 01 & 0,46 \\
\hline Universidade Paranaense (Unipar - PR) & 01 & 0,46 \\
\hline Centro Universitário Adventista de São Paulo (Unasp - SP) & 01 & 0,46 \\
\hline Faculdade Meridional (IMED - RS) & 01 & 0,46 \\
\hline Faculdade Anhanguera de Passo Fundo (FAPF - RS) & 01 & 0,46 \\
\hline Faculdades do Centro do Paraná (UCP - PR) & 01 & 0,46 \\
\hline Universidade Federal de São Carlos (Ufscar - SP) & 01 & 0,46 \\
\hline Universidad Autonoma de Sinaloa (México) & 01 & 0,46 \\
\hline Centro Universitario de Ciencias Económico Administrativas (México) & 01 & 0,46 \\
\hline Universidade Federal do Amazonas (UFAM -AM) & 01 & 0,46 \\
\hline Faculdade Reges Osvaldo Cruz (Feocruz - SP) & 01 & 0,46 \\
\hline Universidade Federal do Mato Grosso do Sul (UFMS - MS) & 01 & 0,46 \\
\hline Universidade Beira Interior (Portugal) & 01 & 0,46 \\
\hline Universidade Potiguar (UnP-RN) & 01 & 0,46 \\
\hline Universidade de Fortaleza (UnF - CE) & 01 & 0,46 \\
\hline Universidade Federal do Rio Grande do Norte (UFRN - RN) & 01 & 0,46 \\
\hline Universidade FUMEC (FUMEC-MG) & 01 & 0,46 \\
\hline Centro Universitário UNA (UNA-MG) & 01 & 0,46 \\
\hline Universidade Antônio Carlos (Unipac - MG) & 01 & 0,46 \\
\hline Faculdade de Administração e Artes de Limeira (FAAL-SP) & 01 & 0,46 \\
\hline
\end{tabular}




\begin{tabular}{llc}
\hline $\begin{array}{l}\text { Instituição } \\
\text { com artigos } \\
\text { publicados }\end{array}$ & $\begin{array}{l}\text { Número de vezes que a } \\
\text { instituição participou com } \\
\text { artigos publicados }\end{array}$ & $\begin{array}{c}\text { f(\%) } \\
\text { Universidade Federal do Espírito Santo (UFES - SP) }\end{array}$ \\
Université Pierre Mendès (França) & 01 & 0,46 \\
Complexo de Ensino Superior de Santa Catarina (Cesusc - SC) & 01 & 0,46 \\
Universidade Braz Cubas (UBC - SP) & 01 & 0,46 \\
Universidade Federal Rural do Semi-árido (Ufersa - RN) & 01 & 0,46 \\
Faculdade Cenecista de Capivari (Facecap - SP) & 01 & 0,46 \\
Centro Universitário Ritter dos Reis (UniRitter - RS) & 01 & 0,46 \\
Universidade do Grande Rio (Unigranrio - RJ) & 01 & 0,46 \\
Universidad Nacional de La Plata (Argentina) & 01 & 0,46 \\
Universidade Pernambuco (UPE - PE) & 01 & 0,46 \\
Universidade Tecnológica Federal do Paraná (Utfpr - PR) & 01 & 0,46 \\
Escola Superior de Administração, Direito e Economia (Esade - RS) & 01 & 0,46 \\
Faculdade de Tecnologia de Jaboticabal (FATEC Jaboticabal - SP) & 01 & 0,46 \\
Universidad de Zaragoza (Espanha) & 01 & 0,46 \\
\hline
\end{tabular}

Fonte: Elaborada pelas autoras.

A seguir comenta-se sobre a entrevista realizada com o editor chefe da RAUNIMEP com o objetivo de complementar informações sobre o estudo.

\subsection{ENTREVISTA COM O EDITOR CHEFE DA RAUNIMEP}

Foi realizada uma entrevista semiestruturada com o editor chefe da RAUNIMEP o qual também foi seu editor acadêmico até o ano de 2007, quando assumiu a editoração.

Em 2007 o editor adotou o Open Journal Systems (OJS), que é um software desenvolvido pela Universidade British Columbia para sistematizar os trabalhos de submissão e avaliação dos artigos da RAUNIMEP. No Brasil este sistema foi traduzido e customizado pelo Instituto Brasileiro de Informação em Ciência e Tecnologia (IBICT) e recebe o nome de Sistema Eletrônico de Editoração de Revistas - SEER.

Atualmente, a revista recebe de 8 a 10 artigos por mês, gerando uma média de 108 artigos por ano, sendo que ocorre um índice de $80 \%$ de rejeições já na primeira avaliação, pois a revista prioriza e preza por artigos de excelência, que tenham contribuição para o atual cenário empresarial.

$\mathrm{O}$ editor cita também que as principais mudanças na trajetória histórica da revista, até setembro/2012 foram à implementação do OJS, a padronização conforme os critérios de cada estrato da CAPES e a qualificação Qualis CAPES de B5 para B3.
O professor avalia a revista como uma revista genérica e ampla que visa publicar artigos de várias áreas da administração e que tem crescido no cenário nacional. Atualmente ela está em um estágio mediano de classificação, porém tem um grande potencial para ser uma revista de destaque e de impacto nacional.

$\mathrm{O}$ maior problema enfrentado pelo periódico ocorre por parte do processo de avaliação pois, nem todos os avaliadores e editores mostram-se adequadamente preparados para este trabalho. Alguns têm dificuldades de cumprirem prazos e fazem entregas de pareceres frágeis. Para um artigo ser publicado na RAUNIMEP ele tem um prazo médio de 6 a 8 meses, pois ele passa por todo um processo editorial e uma blind-review (são dois avaliadores por artigo).

Os pontos fortes da revista que o editor-chefe destacou são: edição e normatização; segue a política de boas práticas editorais da ANPAD; têm crescido no cenário nacional; equilíbrio nos temas e regiões de origem, contribuição de instituições nacionais e internacionais.

O ponto limitante é o processo editorial e avaliação dos artigos, pois é um processo que exige muita responsabilidade e alguns avaliadores retardam este procedimento por demorarem na entrega do parecer e, esta realidade implica em gargalos e pode comprometer o fluxo do periódico.

As perspectivas futuras que o editor-chefe aspira é o crescimento no cenário nacional e elevar a qualificação da revista para A2. 
Ele cita que as últimas conquistas que a revista obteve foram à indexação da EBSCO e da REDALYC. Em novembro de 2012 o periódico passou por mais uma avaliação e subiu para B2 por conta dessas indexações.

Nas considerações finais da entrevista o editor chefe da RAUNIMEP destacou ainda a importância de manter o nível de confiança do seu público e estar adequada ao processo Qualis CAPES de produção científica nos programas de Pós-graduação Strito Sensu.

Quando da finalização do presente artigo ocorreu a reclassificação da RAUNIMEP de B3 para B1, conforme almejada pelo seu editor chefe, porém, para as próximas avaliações pretende-se chegar ao conceito A-2 e posteriormente, ao A-1.

\section{CONSIDERAÇÕES FINAIS}

Este artigo teve como objetivo analisar a produção bibliográfica do periódico RAUNIMEP no período de setembro de 2003 a agosto de 2012. Para esta finalidade, realizou-se pesquisa em dados secundários por meio de levantamento dos 217 artigos publicados neste período. Integrou o processo de coleta de dados uma entrevista semiestruturada desenvolvida com o editor chefe do período.
Os dados coletados nos permitem confirmar a importância da RAUNIMEP para o Programa de Pós-Graduação em Administração da Unimep, bem como para a atividade de pesquisa neste nível.

Destaca-se o processo histórico bem-sucedido que a revista vem desenvolvendo, pela obtenção do OJS/ SEER e pelo trabalho sério e rigoroso do seu editor chefe e equipe de editores e avaliadores.

Os resultados obtidos em curto período de tempo culminaram com as indexações da RAUNIMEP no EBSCO e REDALYC, apontando para a recente conquista que foi a reavaliação no sistema Qualis CAPES para o nível B2.

Pode-se considerar que a RAUNIMEP é um caso de sucesso de um periódico científico eletrônico nacional demostrando projeção internacional pela participação de diferentes pesquisadores estrangeiros.

Em 2014, a RAUNIMEP, encontra-se indexada no Sumarios.org, Directory of Open Acess Journals, Redalyc.org, Latindex. Ulrichs periodicals directory, Ibict Instituto Brasileiro de Informação em Ciência e Tecnologia.

A presente pesquisa limita-se a análise especifica da RAUNIMEP, sugere-se que novas pesquisas sejam realizadas com outros periódicos indexados por classificação, A, B, ou C, uma vez que os critérios de avaliação dos periódicos estão em alterações ano a ano.

\section{REFERÊNCIAS}

BRADFORD, S. C. Sources of information on specific subjects. Egineering, [s.1.], v.137, p. 85-86, 1934.

BIRENE, Biblioteca Regional de Medicina. Fator de Impacto. Centro Latino-Americano e do Caribe de Informação em Ciências da Saúde. Disponível em: <http://bc.bireme.br/f_impacto.htm>. Acesso em: 18 set. 2012.

CAPES, COORDENAÇÃO DE APERFEIÇOAMENTO DE PESSOAL DE NÍVEL SUPERIOR. Administração, Ciências Contábeis e Turismo. Disponível em: <http://www.capes.gov.br/component/ content/article/44-avaliacao/4649-administracao-ciencias-contabeis-e-turismo>. Acesso em: 11 set. 2012.

História e Missão. Disponível em: <http://www.capes.gov.br/sobre-a-CAPES/historia-e-missao>. Acesso em: 11 mar. 2014.

Qualis Periódicos. Disponível em: <http://www.capes.gov.br/sobre-a-CAPES/historia-e-missao>. 
Acesso em: 11 mar. 2014.

Bem Vindo ao WebQualis. Disponível em: $<$ http://qualis.capes.gov.br/webqualis/principal.seam>. Acesso em: 11 mar. 2014.

CHIZZOTTI, A. Pesquisas em ciências humanas e sociais. São Paulo: Cortez, 1991.

FERnANDES, W. R.; CENDÓN, B. V. Portal de Periódicos da CAPES: proposta de um modelo de estudo bibliométrico para avaliação da coleção. Disponível em: < http://www2.capes.gov.br/rbpg/images/stories/ downloads/RBPG/Vol.7_13/5_Artigo.pdf $>$. Acesso em: 11 mar. 2014.

GIL, A. C. Métodos e Técnicas de Pesquisa Social. 5 ed. São Paulo: Atlas, 1999.

Como elaborar projetos de pesquisa. 4 ed. São Paulo: Atlas, 2002.

LOTKA, A. J. The frequency of distribuition of scientific productivity. Journal of the Washington Academy of Sciences, v. 16, n.12, p. 317-323, 1926.

MUELLER, S. P. M. A ciência, o sistema de comunicação científica e a literatura científica. In: CAMPELLO, B. S.; CENDÓN, B. V.; KREMER, J. M. (Org.) Fontes de informação para pesquisadores e profissionais. Belo Horizonte: Editora UFMG, 2000a.

. O periódico científico. In: CAMPELLO, B. S.; CENDÓN, B. V.; KREMER, J. M. (Org.). Fontes de informação para pesquisadores e profissionais. Belo Horizonte: Editora UFMG, $2000 \mathrm{~b}$.

OLIVEIRA, J. C. Estudo bibliométrico das publicações de custos em enfermagem no período de 1966 a 2000. (Dissertação)- Escola de Enfermagem, Universidade de São Paulo, São Paulo, 2001.

PLATAFORMA SUCUPIRA, Periódicos Qualis. Disponível em: <https://sucupira.capes.gov.br/sucupira/ public/consultas/coleta/veiculoPublicacaoQualis/listaConsultaGeralPeriodicos.jsf >. Acesso em: 20 abr. 2016.

RAUNIMEP, REVISTA DE ADMINISTRAÇÃO DA UNIMEP. Missão. Disponível em: <http://www. raunimep.com.br/ojs/index.php/regen/index>. Acesso em: 19 ago. 2012.

Edições Anteriores. Disponível em: <http://www.raunimep.com.br/ojs/index.php/regen/issue/ archive?issuesPage=2>. Acesso em: 23 set. 2012. mar. 2014.

Missão. Disponível em: <http://www.raunimep.com.br/ojs/index.php/regen/index>. Acesso em: 11

Edições Anteriores. Disponível em: <http://www.raunimep.com.br/ojs/index.php/regen/issue/ archive?issuesPage=2>. Acesso em: 11 mar. 2014.

VANTI, N. A. P. Da bibliometria à webometria: uma exploração conceitual dos mecanismos utilizados para medir o registro da informação e a difusão do conhecimento. Ciência da Informação, Brasília, v. 31, n. 2, p. 152-162, 2002.

WEBQUALIS, Classificação da Produção Intelectual. Disponível em: <http://www.capes.gov.br/avaliacao/ instrumentos-de-apoio/classificacao-da-producao-intelectual>. Acesso em: 23 abr. 2014. 